EPJ manuscript No.

(will be inserted by the editor)

\title{
A Review of Searches for R-parity-violating SUSY
}

\author{
Christian Schwanenberger ${ }^{a}$ b \\ Deutsches Elektronen-Synchrotron (DESY), Notkestr. 85, D-22607 Hamburg \\ Received: date / Revised version: date
}

\begin{abstract}
Searches for pair and single production of supersymmetric particles under the assumption that $R$-parity is violated via a single dominant coupling are presented. A subset of the most recent results from LEP, Tevatron and HERA is selected. The data are in agreement with the Standard Model expectation. Limits on the production cross sections and the masses of supersymmetric particles are derived.
\end{abstract}

PACS. 11.30.Pb Supersymmetry - 04.65.+e Supergravity - 12.60.Jv Supersymmetric models

\section{Introduction}

$R$-parity $\left(R_{p}\right)$ [1] is a discrete multiplicative symmetry. It can be written as $R_{p} \equiv(-1)^{3 B+L+2 S}$. Here $B(L)$ denote the baryon (lepton) number and $S$ the spin of a particle. For Standard Model (SM) particles $R_{p}=+1$, while $R_{p}=-1$ for their supersymmetric partners. The most general superpotential with the minimal field content of the supersymmetric SM contains the trilinear $R_{p}$-violating terms ${ }^{1}$ :

$$
W_{\not R_{p}}=\lambda_{i j k} L_{i} L_{j} \bar{E}_{k}+\lambda_{i j k}^{\prime} L_{i} Q_{j} \bar{D}_{k}+\lambda_{i j k}^{\prime \prime} \bar{U}_{i} \bar{D}_{j} \bar{D}_{k}
$$

where $L_{i, j}\left(Q_{j}\right)$ are the lepton (quark) doublet superfields, $\bar{D}_{j, k}, \bar{U}_{i}\left(\bar{E}_{k}\right)$ are the down-like and up-like quark (lepton) singlet superfields, $\lambda, \lambda^{\prime}\left(\lambda^{\prime \prime}\right)$ are Yukawa couplings which violate $L(B)$ conservation $^{2}$ and $i, j, k=1,2,3$ are the generation indices $\left[2 .{ }^{3}\right.$ Hierarchies in $\not R_{p}$ couplings are supposed as for Yukawa couplings generating fermion masses. Thus, here one assumes that a single coupling dominates.

$\not R_{p}$ opens a new scenario in supersymmetric searches complementary to the $R_{p}$-conserving ones. The conservation of $R_{p}$ implies that sparticles can only be produced in pairs. Their decay ends in final states containing the lightest supersymmetric particle (LSP) which is stable. The phenomenological consequences of $R_{p}$ are that single sparticle production is possible and that the LSP decays into SM fermions. The latter can lead to final states with a large number of leptons or jets.

\footnotetext{
a Talk presented at the International Europhysics Conference on High Energy Physics EPS 2003, Aachen, July 2003.

b Send offprint requests to: schwanen@mail.desy.de

1 Bilinear terms are not considered in this report.

2 Fast proton decay is suppressed if $\Delta L \neq 0$ and $\Delta B \neq 0$ operators are not simultaneously present.

${ }^{3}$ Here $45 \not R_{p}$ couplings are introduced. If spontaneous $\not R_{p}$ is considered, there are three additional terms.
}

In the analyses presented here both single sparticle production via a $\not R_{p}$ coupling and pair production of sparticles via a $R_{p}$-conserving coupling are investigated. The study of pair-produced sparticles allows one to constrain sparticle masses, independently of whether $R_{p}$ is conserved. In the analyses presented here two types of sparticle decays are considered. First, the direct decay into two SM fermions via a $\not R_{p}$ vertex and, second, the indirect decay via $R_{p}$-conserving interactions in cascades down to the LSP, which then decays via a $\not R_{p}$ vertex.

\section{LEP searches}

In $e^{+} e^{-}$collisions sparticle pair production has been investigated at center of mass energies up to $209 \mathrm{GeV}$. No deviation from the SM was found. Limits are derived on cross-sections and couplings $\lambda, \lambda^{\prime}, \lambda^{\prime \prime}$. The limits on sparticle masses are as stringent as in the $R_{p}$-conserving analyses. Furthermore, exclusion plots in the Minimal Supersymmetric Standard Model (MSSM) are produced. Details can be found in [3. As an example, exclusion limits in a Constrained MSSM (CMSSM) for $\widetilde{\nu}_{e}$ pair production for $\widetilde{\nu}_{e}$ decaying via a $\lambda$ coupling (direct) into 4 leptons $\left(e^{+} e^{-} \rightarrow \widetilde{\nu} \widetilde{\nu} \rightarrow \ell^{-} \ell^{+} \ell^{+} \ell^{-}\right)$or via a gauge coupling (indirect) producing 4 leptons and missing energy $\left(e^{+} e^{-} \rightarrow \widetilde{\nu} \bar{\nu} \rightarrow \nu \widetilde{\chi}_{1}^{0} \bar{\nu} \widetilde{\chi}_{1}^{0} \rightarrow \nu\left(\stackrel{(-)}{\nu} \ell^{+} \ell^{-}\right) \bar{\nu}\left(\stackrel{(-)}{\nu} \ell^{+} \ell^{-}\right)\right)$ are shown in Fig. 1] This analysis was performed by the OPAL experiment using a total integrated luminosity of $610 \mathrm{pb}^{-1}$.

\section{Tevatron searches}

A search for pair production of stop quarks $\left(\widetilde{t}_{1}\right)$ using $106 \mathrm{pb}^{-1}$ of $p \bar{p}$ collisions at $\sqrt{s}=1.8 \mathrm{TeV}$ has been performed by CDF 4] (Tevatron Run I). In the investigated 


\section{OPAL}

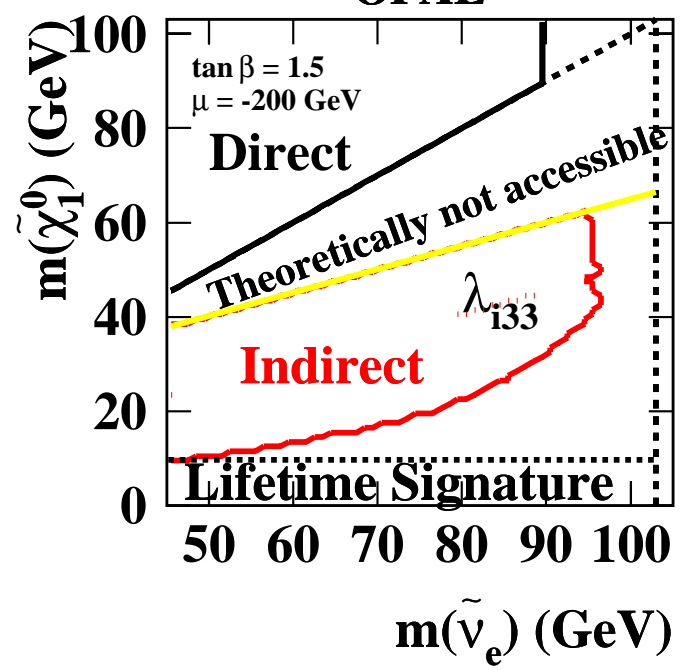

Fig. 1. CMSSM exclusion region for $\widetilde{\nu}_{e} \widetilde{\nu}_{e}$ production in the $\left(m_{\widetilde{\nu}_{e}}, m_{\widetilde{\chi}_{1}^{0}}\right)$ plane at $95 \%$ confidence level (CL) for a $\lambda$ coupling

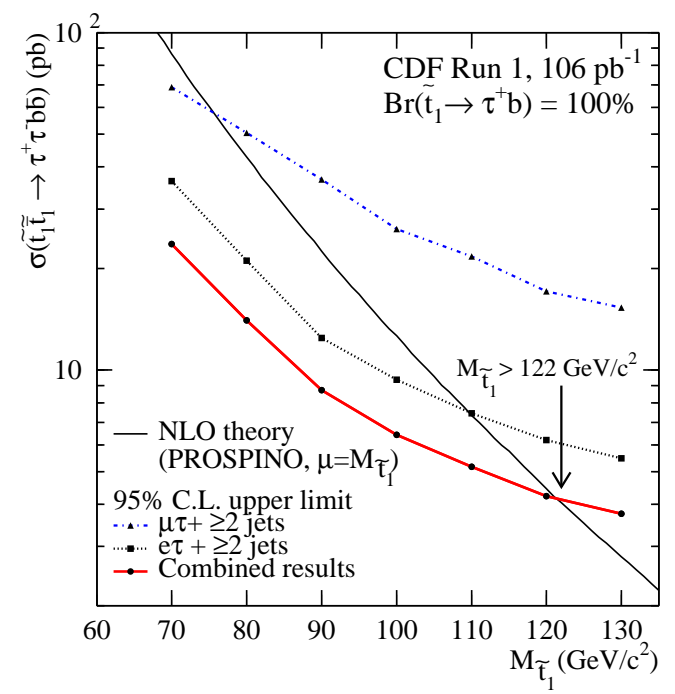

Fig. 2. Upper limits at $95 \% \mathrm{CL}$ on cross section for $\widetilde{t}_{1} \overline{\widetilde{t}}_{1}$ production compared to the NLO calculations

mode each $\widetilde{t}_{1}$ decays into a $\tau$ lepton and a $b$ quark. The search demands events with two $\tau$ 's, one decaying leptonically $(e$ or $\mu$ ) and one decaying hadronically, and two jets. No candidate event passes the final selection criteria. Fig. [2 shows upper limits on the cross section for a branching ratio of $100 \%$ for the $e, \mu$ and combined channels, along with the NLO prediction of the production cross sections. A lower limit of $\approx 122 \mathrm{GeV}$ for the $\widetilde{t}_{1}$ mass has been derived. Since the analysis does not distinguish the quark flavors in jet reconstruction, these results are equally valid for any $\lambda_{33 k}^{\prime}(k=1,2,3)$ coupling.

\section{HERA searches}

The ep collider HERA is well suited to search for resonant squark production via $\not_{p}$ interactions. With an ini-

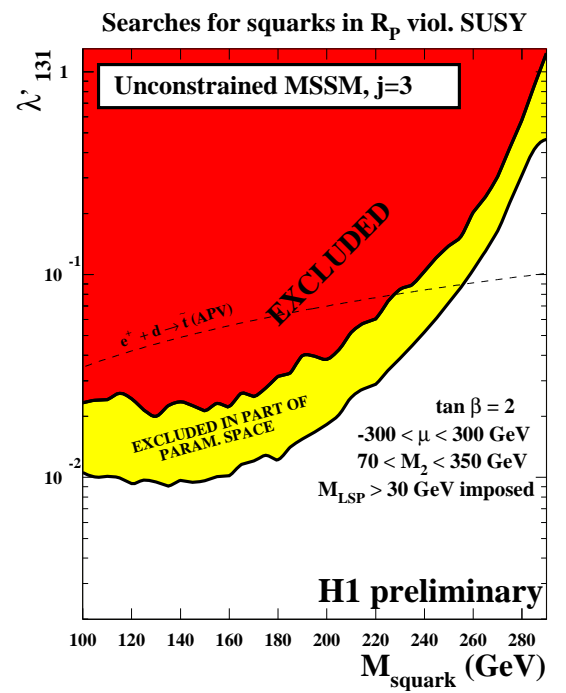

Fig. 3. Upper limits at $95 \%$ CL for the coupling $\lambda_{131}^{\prime}$ as a function of the squark mass, in the "phenomenological" MSSM

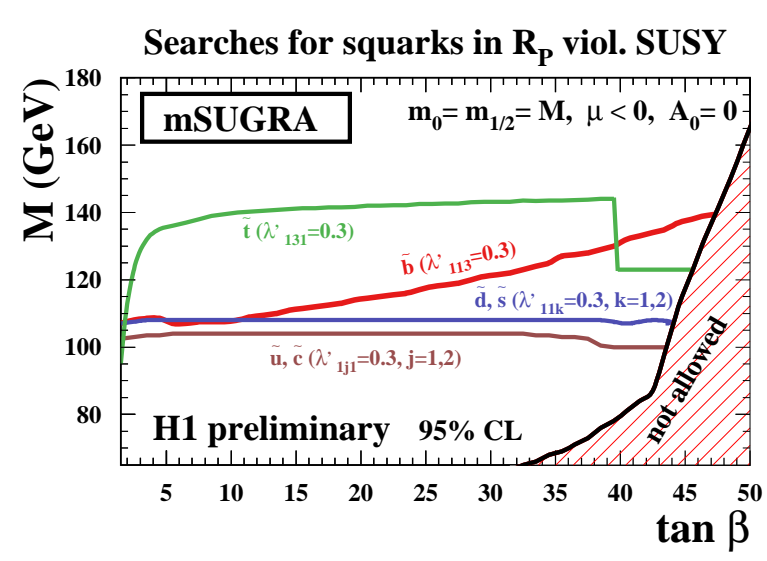

Fig. 4. Constraints at $95 \% \mathrm{CL}$ obtained in the mSUGRA model, assuming $\lambda_{1 j k}^{\prime}=0.3(j, k=1,2,3)$. The areas below the curves are excluded

tial $e^{+}$beam the sensitivity is highest to couplings $\lambda_{1 j 1}^{\prime}$ $(j=1,2,3)$, where mainly $\widetilde{u}_{L}^{j}$ squarks are produced. In contrast, with an initial $e^{-}$beam HERA is most sensitive to couplings $\lambda_{11 k}^{\prime}(k=1,2,3)$ and can mainly produce $\widetilde{d}_{R}^{k}$ squarks.

The resonant production of single $\widetilde{u}_{L}^{j}$ and $\widetilde{d}_{R}^{k}$ squarks has been investigated by $\mathrm{H} 1$ using data taken at a center of mass energy of $\sqrt{s}=319 \mathrm{GeV}$ corresponding to an integrated luminosity of $64 \mathrm{pb}^{-1}$ for $e^{+} p$ collisions and $13.5 \mathrm{pb}^{-1}$ for $e^{-} p$ collisions [5]. No deviation from the SM prediction has been observed. Upper bounds on the production cross section are derived by combining all channels from direct and indirect squark decays. In SUSY models inspired by the Minimal Supersymmetric Standard Model (MSSM), the channels considered in this analysis cover $\approx 100 \%$ of all possible squark decay modes. These bounds are translated into constraints on the parameters of SUSY models. 


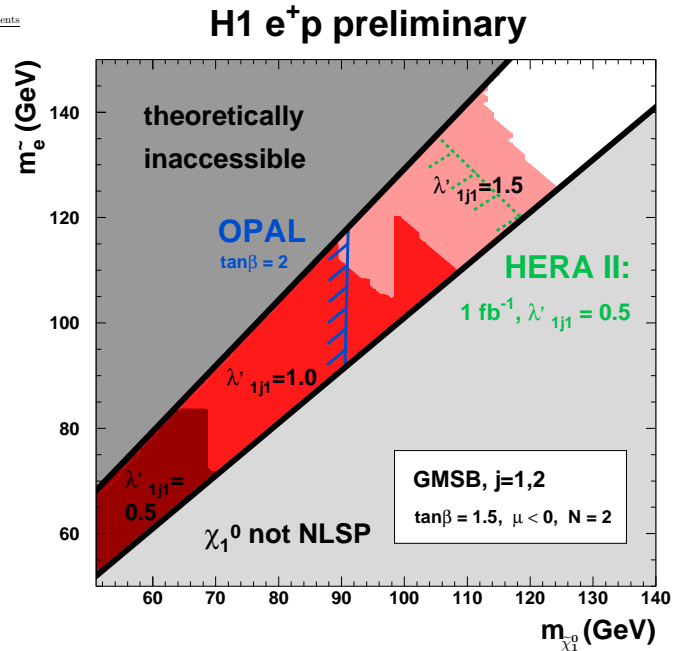

Fig. 5. Excluded regions at $95 \% \mathrm{CL}$ in the GMSB model dependent on selectron and neutralino mass assuming $\lambda_{1 j 1}^{\prime}=0.5$ (dark red), 1.0 (red), 1.5 (light red) $(j=1,2)$

Fig. [3] shows upper limits on the Yukawa coupling $\lambda_{131}^{\prime}$ as a function of the $\widetilde{t}_{L}$ mass. These are obtained in a "phenomenological" MSSM, where the gaugino masses are related to each other while the sfermion masses are free. A scan of the parameter space is performed, which shows that the obtained limits do not depend strongly on the model parameters. The limits obtained on the coupling $\lambda_{131}^{\prime}$ (and on $\lambda_{121}^{\prime}$ - not shown here) extend beyond the indirect bounds from low energy experiments. For a coupling of electromagnetic strength $\left(\lambda^{\prime}=0.3\right)$ stop masses up to $\approx 270 \mathrm{GeV}$ are excluded. E.g. the future sensitivity of the Tevatron Run II experiments on light stop quarks might be around $200-250 \mathrm{GeV}$, depending on the main decay modes of the stop. Thus, a reasonably large coupling $\lambda_{131}^{\prime}$ would provide an interesting discovery potential for the stop at HERA II with the much larger integrated luminosity expected within the next few years.

Constraints obtained in the framework of the minimal Supergravity (mSUGRA) model have also been derived. Here, a common mass $m_{0}\left(m_{1 / 2}\right)$ is assumed for the scalars (gauginos) at the Grand Unification scale. Lower limits for $m_{0}=m_{1 / 2}=M\left(\lambda^{\prime}=0.3\right)$ are presented in Fig. 目as a function of $\tan \beta$, the ratio of the vacuum expectation values of the two neutral scalar Higgs fields. The single production of all 6 squark flavors is considered.

In another $\mathrm{H} 1$ analysis, neutralino $\left(\widetilde{\chi}_{1}^{0}\right)$ production via $\not R_{p} t$-channel selectron exchange $\left(e^{+} p \rightarrow \widetilde{\chi}_{1}^{0}+1\right.$ jet $)$ has been studied in Gauge Mediated Supersymmetry Breaking (GMSB) scenarios where the slepton masses are usually much lower than the squark masses 6 . The gravitino is the LSP, while the neutralino is assumed to be the nextto-lightest supersymmetric particle (NLSP). The prompt decay of the neutralino into a photon and a gravitino is analyzed, which leads to prominent event signatures with a photon and large missing transverse momentum. No significant deviation from the SM has been found using $64 \mathrm{pb}^{-1}$ of $e^{+} p$ collisions at $\sqrt{s}=319 \mathrm{GeV}$.

In Fig. [5 excluded regions in the $\left(m_{\tilde{e}}, m_{\tilde{\chi}_{1}^{0}}\right)$ plane for different values of the $R_{p}$ coupling are presented. For se-

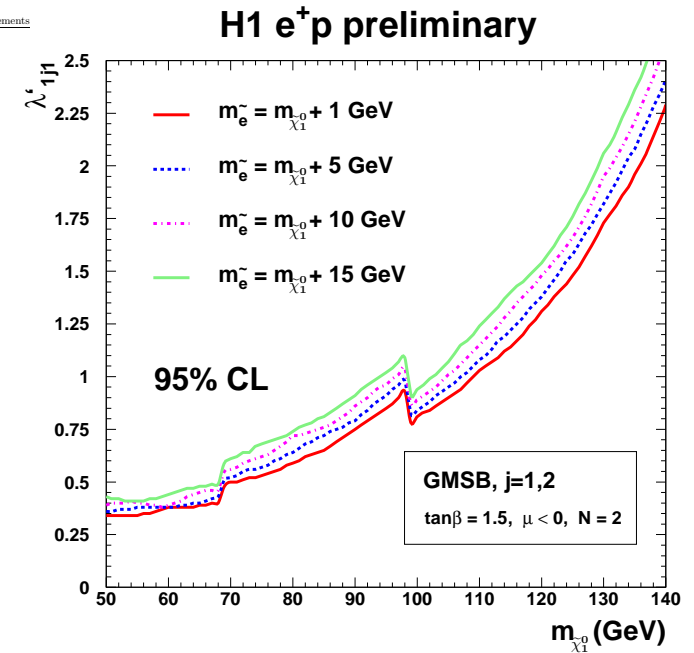

Fig. 6. Upper limits at $95 \%$ CL for the coupling $\lambda_{1 j 1}^{\prime}(j=1,2)$ as a function of the neutralino mass for various differences between the selectron and neutralino masses

lectron masses very close to the neutralino mass and for $\lambda_{1 j 1}^{\prime}=1.0$, neutralino masses up to $\approx 108 \mathrm{GeV}$ can be excluded. For moderate values of $\lambda_{1 j 1}^{\prime}$, the region excluded by the OPAL [7] analysis of $e^{+} e^{-} \rightarrow \widetilde{\chi}_{1}^{0} \widetilde{\chi}_{1}^{0} \rightarrow \gamma \tilde{G} \gamma \tilde{G}$ in a $R_{p}$-conserving SUSY scenario can be extended. In Fig. [6] upper limits on $\lambda_{1 j 1}^{\prime}(j=1,2)$ are given as a function of $m_{\tilde{\chi}_{1}^{0}}$ for various assumptions for the difference between selectron and neutralino mass. These are the first limits derived for $\lambda_{121}^{\prime}$ which are independent of squark masses.

\section{Conclusions and prospects}

The violation of R-parity has inspired new interesting scenarios for SUSY searches. Many different channels for single or pair production of SUSY particles in a $R_{p}$ scenario have been investigated at LEP, Tevatron and HERA. However, no deviation from the SM has been observed. The derived limits from $R_{p}$ searches are comparable with the $R_{p}$-conserving limits. Thus, searches for $R_{p}$ deliver important contributions to constraints on SUSY models. Further interesting results can be expected at Tevatron Run II, HERA II and future colliders.

\section{References}

1. P. Fayet, Phys. Lett. B 69 (1977) 489; G. Farrar and P. Fayet, Phys. Lett. B 76 (1978) 575.

2. H. Nilles, Phys. Rept. 110 (1984) 1; H. E. Haber and G. L. Kane, Phys. Rept. 117 (1985) 75.

3. OPAL Collab., submitted to Eur. Phys. J. C, hep-ex/0310054 ALEPH Collab., Eur. Phys. J. C 25 (2002) 1; ALEPH Collab., Eur. Phys. J. C 31 (2003) 1; DELPHI Collab., Eur. Phys. J. C 28 (2003) 15; DELPHI Collab., Abstract 337, submitted to EPS03, Aachen.

4. CDF Collab., hep-ex/0305010

5. H1 Collab., Abstract 101, submitted to EPS03, Aachen.

6. H1 Collab., Abstract 676, submitted to EPS03, Aachen.

7. OPAL Collab., Phys. Lett. B 501 (2001) 12. 